and thirteen pieces of bone, varying from the size of a threepenny-piece to sixpence, were removed. The membranes were torn, and one piece of bone, of the size of sixpence, and composed of both plates of the skull, was felt to be almost buried in brain-matter. The point of this was seized by the dissecting forceps and removed. Several pieces of the inner table were removed. Small portions of brain-matter escaped.

The wound was left open and covered with wet lint

The patient expressed himself as feeling easier immediately after the operation. The surface was cold; pupils equal, but not answering readily to light. Hot bottles were ordered to the feet, the patient to be kept perfectly quiet, and the room darkened. Diet to consist of milk and beef-tea. A large saline purgative was given, and five grains of mercury with chalk ordered to be taken every six hours. Pulse 80.

About two hours after the operation the patient said he felt easy and was inclined to sleep ; the surface was warmer, but the pulse was not much quickened, being 90 .

Oct. 2 nd. - Pulse 94 ; respiration 26 ; temperature $99^{\circ}$. Slept well during the night. Free from pain. Skin warm; pupils equally dilated; bowels not open. Ordered to take five grains of mercury pill immediately, to be repeated if necessary. Passed urine. Tongue furred. Evening tem. perature $99^{\circ}$.

3rd.-Pulse 78 ; respiration 20 ; temperature $992^{\circ}$. Passed a good night, only waking to take his powder ; was dreaming a little. No pain whatever in the bead. Bowels opened this morning. Passed urine freely. Pupils dilated, both answering to light. Slight facial paralysis. Speech altered, but not indistinct. Loss of power in right hand rather less. Last evening, after seeing a policeman and two or three strangers, against orders, be did not feel so well, and his speech became more affected. Wound dressed; looking well. Evening temperature $984^{\circ}$.

4th. - Pulse 74 ; respiration 20 ; temperature $98.4^{\circ}$. Did not sleep quite so well as on the previous night, being kept awake by pain over the left side of the head. The pain lasted about an hour, then went. Quite free from pain now. Bowels open once. Speech rather more indistinct. No sickness. Mouth slightly sore from the mercury. Powder to be taken twice a day. Evening temperature $984^{\circ}$.

5 th.-Pulse 64 ; respiration 20 ; temperature $98.4^{\circ}$. Going on weli. Wound looking healtby.

From this date the patient never had a bad symptom, and the temperature was never above normal. On Oct. 20 th the wound was just healed, and all the symptoms of paralysis were gone. On Nov. 8 th he was at work again. On Dec. 10th he called to see me, and he continued quite well.

Flaxton, York,

\section{d 算tirutor}

\section{HOSPITAL PRACTICE,}

\section{BRITISH AND FOREIGN.}

Nulla autem est alia pro certo noscendi via, nisi quamplurimas et morborum et dissectionum historias, tum aliorum, tum proprias collectas habere, et inter se comparare.-Mongagn I De Sed. et Caus. Morb., lib.iv. Procemium.

\section{KING'S COLLEGE HOSPITAL.}

A CASE OF INTCSSUSCEPTION OF THE LARGE INTESTINE; ABDOMINAL SECTION ; DEATH.

(Under the care of Mr. Royes BeLL.)

THE following case is important as illustrating the difficulty that may attend the reduction of an intussusception, even when there are no adhesions.

The patient was originally under the care of Dr. J. Henry Philpot, physician to the St. Pancras and Northern Dispensary, to whom we are indebted for the subjoined notes.

A male child, sixteen months old, was taken by his grandmother to the dispensary on October 21 st. The grandmother stated that the child had been delicate from birth, and had been brought up by hand; and that he had been troubled for a long time with diarrbca. On Oct. 17th the diarrhoa suddenly ceased, but as the child seemed as well as usual, except that he showed less desire for food, no attention was paid to it. When in bed with him on the night of the 18th she was disturbed by his suddenly crying out with pain, and noticed that be had wetted his night-dress. By the morning light she found his night-dress stained with blood, and there was also a clot of dark blood on it of the size of a half-crown. From that time the child became manifestly worse: he was dull and sleepy; refused nearly all food, and mostly brought up the little that he had been coaxed to take. At the same time there was great thirst. The child showed signs of suffering from pain, and his belly swelled: Every now and then he appeared to strain, but the only result was a small quantity of slime and blood.

When first seen (October 21st) the child looked pale and anxious; his limbs were thin aud flabby, but the abdomen was swollen and tympanitic. On deep pressure in the left iliac region, a distinct tumour could be felt extending up into the left lumbar region. Its limits could be clearly mapped out by percussion, and it was found to be about three inches in length, and one inch in breadth. About the anus some blood-stained viscid mucus was found clinging. On digital examination per rectum, no obstruction could be detected, but on gently passing a soft bougie, some resistance was met with about five inches above the anus. The child bore all these manipulations without complaint. Intussusception of the larger bowel was diagnosed. On the following day the child was seen by Mr. Cross, the resident surgeon, who stated that, on rectal examination by the finger, the anus was found to be very lax and the rectiom roomy; about three inches from the anus, the finger met with an obstruction, which was evidently an intussuscepted portion of the bowel, which was felt projecting into the rectum in the form of a soft, rounded tumour, resembling the os uteri in the pregnant state. The finger could not only be passed round the descended gut, between it and the rectum, but was also easily admitted into the orifice of the former. On witbdrawing the finger, it was found smeared with blood and mucus, showing that the intussuscepted mass bled easily. On examination with the rectal speculum, the invaginated portion presented an ash-grey colour. The resisting tumour was still to be felt in the left iliac fossa, and its boundaries could be distinguished on percussion as before. Copious injections of warm soap-and-water were made by Mr. Cross, but without the desired effect of restoring the intussuscepted bowel.

As it was impossible to proceed to more radical treatment at the grandmother's house, the child was admitted into King's College Hospital, under the care of Mr. Royes Bell, who is also surgeon to the dispensary.

On Saturday, the 23rd, six days after the cessation of the diarrboea, the symptoms were in all essential points unchanged, except that the child was more apathetic, the abdomen was more swollen and tympanitic, and there was still less appetite. Vomiting continued, but was by no means frequent or distressing, and the ejecta were inoffensive. It was determined on consultation first to inject air per rectum, and in the event of that expedient proving unsuccessful, to proceed at once to perform abdominal section. A few whiffs of chloroform were given, and a tube was passed up to the orifice of the intussuscepted portion, gentle attempts being made to return the bowel by this means. These attempts were, however, unsuccessful, as was also the injection of air into the lower bowel and into the orifice of the prolapsed bowel.

Abdominal section was then performed. An incision through the skin was made down the median line of the abdomen, commencing one inch above the umbilicus, and extending nearly to the symphysis pubis, and the layers of abdominal wall were divided upon a director. The peritoneum was carefully opened, and the intestines were seen to be much distended with gas, and pink and injected on their surface. It was impossible to prevent some portion of the small intestines from escaping from the wound, but they were immediately covered by a cloth wrung out in warm water. The intussuscepted bowel was readily found, occupying the left iliac and lumbar regions. It was from three to four inches in length, about one inch in diameter, and sausage-shaped, solid to the touch, and dark purple in colour, with distended vessels ramifying upon its surface It was evident that a portion of the descending colon had become intussuscepted in the sigmoid flexure. Attempts were made to reduce it, but though as much traction was 
made as could be safely ventured upon, it conld not be moved. Mr. Bell therefore proceeded to pass threads through the portion of bowel immediately above the intussusception, and then opened it. A quantity of grey fæcal fluid and some gas escaped from the opening, and the former was received upon sponges, none of it being allowed to come into contact with the peritoneum. The escaped bowels were then returned with some difficulty, and the opening in the abdominal wall was closed, the artificial opening in the bowel being sutured to the upper part of the external wound. The child seemed to bear the operation well, but his strength gradually failed, and he died about seven hours after it.

On examination of the body forty hours after death, the thoracic viscera were found quite normal. In the abdominal carity the intestines were glued together by recently formed lympk. The intussuscepted portion was discovered occupying the left region of the abdomen. A part of the transverse colon, the splenic flexure, and part of the descending colon were intussuscepted by the rest of the descending colon and the sigmoid flexure, whose serous coat was ruptured in several places. It was found impossible to reduce the invaginated bowel by using ordinary force. On opening up the sigmoid flexure the intussuscepted portion was seen to be twisted in a somewhat spiral manner. On cutting through the second layer of sigmoid flexure no lymph was found between the serous surfaces, except at the extreme lowest portion of the bend. The portion of bowel project. ing in to the rectum was found deeply congested and moderately hard. The orifice in its centre was elongated, owing to the traction of the mesentery in that portion of the lip of the orifice corresponding to its attachment. A largesized catheter could be passed right through the intussuscepted portion into the normal intestine above it. The stomach and the rest of the intestines were moderately distended with gas; the other abdominal organs were per. fectly natural.

It may be remarked, that from the first there could be no doubt about the diagnosis, but, in any case, the prognosis was almost hopeless, especially after the failure of the milder methods of treatment. It was one of those cases in which $\mathrm{Mr}$. Hutchinson recommends early resort to the operation of abdominal section; but though, as proved at the post-mortem, the adjacent serous coverings of the bowels were not bound together by lymph to any great extent, it was found impossible to reduce the intussusception by traction, either in the operating theatre or in the dead-house. The history of the case-intussusception following on con. stant diarrboa-points to another risk attending the latter condition in young children, and provides an additional argument in favour of arresting it as soon as possible.

\section{NORTH-EASTERN HOSPITAL FOR CHILDREN.}

PROBABLE REDUCTION OF INTUSSUSCEPTION BY COPIOUS INJECTION WITH THE BODY INVERTED.

(Under the care of Mr. WAREN TAY.)

Is tine following cases the value of copious injections, while the pelvis was raised, was well exemplified.

An infant, aged seven months, came under care Sept. 26th, 1874. On the previous morning it was taken ill suddenly, began to strain to pass a motion, seemed in pain, und passed only blood and slime, and was sick. Mr. Butlin and Dr. Dixon, of Victoria-park, saw the child, and suspected that intussusception had occurred. Air was injected with a pair of bellows, but no relief followed. When seen, about thirty hours after the commencement of the symptoms, the child was straining constantly, as if about to pass a motion, and seemed in pain. A finger passed up the rectum encountered some obstruction high up, which felt as if it might be the lower end of an intussusception, but it was so high up that this could not positively be stated to be the case. When withdrawn, the finger was covered with blood and slime. No definite tumour could be felt on the left side through the abdominal wall.

A quantity of lukewarm water was injected into the rectum pile the child was held up by the feet. As large a quantity as possible was injected. The child seemed relieved immediately the fluid was allowed to run out, and on examination by the rectum no sensation was communicated to the finger similar to that felt before the injection. When seen the next day, the child seemed quite lively and well, and had remained so when heard of some time later.

Straining, accompanied by the passage of blood and slime by the rectum, and the presence of blood and slime in the rectum, detected on examination, are so fairly characteristic of the occurrence of intussusception in children, that the diagnosis in this case admits of little doubt. It is confirmed by the probable presence of a bulging within the rectum, and by the great and immediate relief which followed the treatment adopted. It is probably not very material whether air or water be injected, but it is very important to have the patient held up by the heels. Mr. Hutchinson has repeatedly insisted on this. An American physician (Dr. Battey) has lately stated that fluid can be made to gravitate slowly through the ileo-cæcal valve, along the whole tract of the small intestine, through the stomach, and even out of the mouth. However this may be, there can be no doubt that in certain cases of obstruction of the bowels the injection of copious enemata during or followed by inversion of the body, and, if necessary, whilst the patient is under the influence of chloroform, is succeeded by complete relief, even where the symptoms are very acute. A case of this sort came under Mr. Tay's care at the London Hospital in 1872. It is sometimes difficult to ascertain when the injection has been carried far enough in cases of intussusception. A child aged fifteen months was taken to the North-Eastern Hospital on June 30th, 1870, suffering from an intussusception of twelve hours' duration. It could be felt just within the anus. The child was sick, had passed blood and mucus, and seemed in great pain. The invagination could not be replaced with the finger, but when warm water was freely injected, the tumour which had been felt slipped out of reach, and great relief was apparently afforded the child. It was considered that the intussusception had been reduced. The child, however, continued to pass only bloody slime, and when seen the following day some tumour could be detected as high up as the finger would reach. A copious injection was again thrown up, while chloroform was given, and the child was inverted, but no change was produced in the symptoms. The child lived for six days altogether, and at the post-mortem an exceedingly short invagination of the transverse into the descending colon was found. There were no traces of peritonitis, and the invagination was easily reducible. Probably more perseverance at first, after the injection had seemed successful, would have completed the release of the bowel. This case showed the fallacy of being deterred from operative procedures on the supposition that peritonitis must necessarily have set in and that the parts were glued together.

\section{ST. BARTHOLOMEW'S HOSPITAL.}

A VERY UNUSUAL CONGENITAL DEFORMITY. (Under the care of Mr. Thomas Smith.)

AuICE T-, aged five months, was admitted into Lucas ward Sept. 20 th, 1875 . The patient was a healthy, wellnourished child. She has a well-formed mouth in the usual situation. On the left side, below the angle of the mouth,

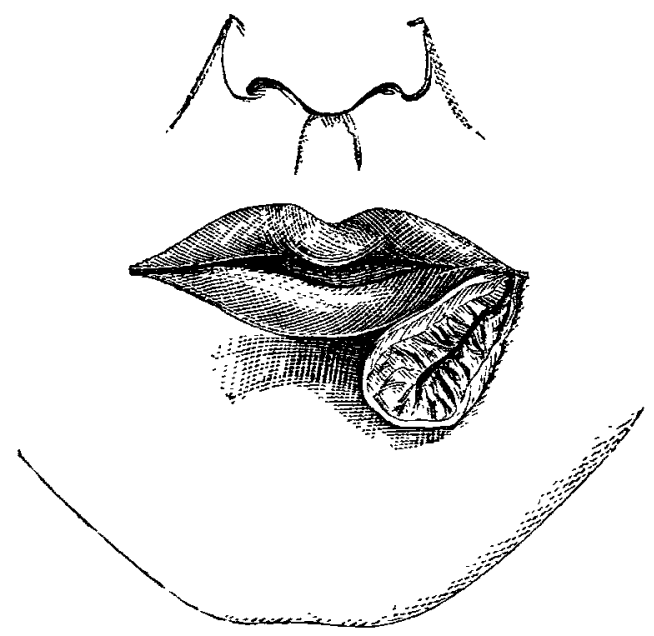

was a second orifice, surrounded by mucous membrane and a sphincter muscle. Its position and size were as represented in the accompanying woodcut. There was a small 